\section{Working on the brain gang(lioside)}

\section{By Lev Osherovich, Senior Writer}

Canadian researchers have found that injecting ganglioside GM1, a naturally occurring lipid, into the brains of mice with Huntington's disease improves motor function and slows disease progression. ${ }^{1}$ The team is in talks with an undisclosed company to license the findings.

Ganglioside GM1 (GM1) is a complex, polysaccharide-bearing lipid that helps hold together lipid rafts, which are dense regions of the plasma membrane that harbor many membrane-associated proteins. The lipid plays a role in neuron differentiation, neurite extension and axon growth during brain development. In mature brains GM1 also modulates multiple signaling mechanisms involved in synaptic functioning.

In 2010, a team led by Simonetta Sipione, assistant professor of pharmacology at the University of Alberta, reported that mice with HD and fibroblasts from patients with HD had lower levels of GM1 than healthy controls. ${ }^{2}$ Also that year, a separate group found that postmortem brain samples from HD patients had abnormally low GM1 levels. $^{3}$

Now, Sipione's team has shown that restoring GM1 levels has a therapeutic effect in mice with HD.

The group infused the lipid into the brain ventricles of HD mice for 28 days using a peristaltic pump. This treatment restored GM1 in the surrounding brain tissue to levels comparable to those in wild-type mice.

Mice treated with GM1 had improved motor function and higher levels of both phosphorylated huntingtin (Htt) and protein phosphatase 1 regulatory inhibitor subunit 1B (Ppp1r1b; Darpp-32) compared with mice given vehicle. Low levels of phosphorylated HTT and DARPP-32 are predictors of HD progression.

Results were reported in the Proceedings of the National Academy of Sciences.

"The mice we used already had a significant motor phenotype, and we were able to reverse that," said Sipione.

She said GM1 immediately improved the behavior of mice that were already sick. "Even after discontinuation of the treatment, motor function in the treated mice was much better than in untreated mice for up to two weeks," Sipione added.

One outstanding question is whether treatment with GM1 could prevent the suspected cause of HD-the misfolding of mutated HTT. Sipione said GM1 may be disease modifying. She noted that in patients with HD, "neurological symptoms appear before there is loss of neuronal tissues," so GM1 therapy could be useful early in the disease's course.
Her next step is to examine the effect of GM1 on Htt aggregation and neuron loss in a different mouse model with a more severe and rapidly developing form of the disease.

Paul Muchowski, associate investigator at the Gladstone Institute of Neurological Disease and associate professor of neurology at the University of California, San Francisco, said the effect of GM1 on misfolded Htt is still unknown and that it remains unclear whether GM1 would truly reverse the disease or instead treat its symptoms.

"Whatever GM1 is doing, it's acting quite rapidly when you dose the mice, and the effect goes away when you stop dosing," said Muchowski. "This suggests that GM1 would be more of a symptomatic therapy" than a disease-reversing treatment.

The predominant therapeutic strategies for $\mathrm{HD}$ aim to alleviate symptoms by increasing neurotransmitter levels or providing trophic factors to promote neuronal survival. The only FDA-approved HD drug is Xenazine tetrabenazine, a vesicular monoamine transporter 2 (VMAT2; SLC18A2) inhibitor from $\mathbf{H}$. Lundbeck A/S.

The next most advanced HD compound is Huntexil pridopidine, a dopamine stabilizer from NeuroSearch $\mathbf{A} / \mathbf{S}$ that is in Phase III testing.

\section{General manager}

Sipione thinks GM1 may work by increasing the activity of multiple signaling pathways. "GM1 modulates the action of various receptors, but I don't think it has very much specificity for any particular pathway-it has pleiotropic effects," she said.

Steven Finkbeiner, senior investigator and associate director of the Gladstone Institute of Neurological Disease and professor of neurology and physiology at the University of California, San Francisco, said Sipione's study was in line with previous evidence of lipid abnormalities in HD.

"There's a line of research in HD and other neurodegenerative diseases that has looked at lipids, though the details are still pretty murky," said Finkbeiner.

He added that previous studies have shown abnormally low levels of cholesterol in HD brains. "Both cholesterol and GM1 are components of lipid rafts," noted Finkbeiner. He said normal HTT is thought to interact with lipid rafts and that this interaction may somehow be compromised by $\mathrm{HD}$-associated mutations.

Thus, one possible mechanism to explain Sipione's findings is that raising GM1 levels alters the composition of lipid rafts and prevents the subcellular mislocalization of mutant $\mathrm{Htt}$, thus preventing the protein's disease-causing effects.

To uncover the mechanism of GM1's effect on HTT, Finkbeiner
“It's always been a question in PD and other neurodegenerative disorders - is there a systemic defect in lipid metabolism, and does this contribute to cell death?" - Jay Schneider LZ Therapeutics Inc. suggested further cell culture and microscopy studies in primary human neuron models of HD.

Jay Schneider, professor of pathology, anatomy and cell biology at Thomas Jefferson University, said defects in lipid metabolism may 


\section{ANALYSIS}

be a common feature of other neurodegenerative diseases, including Parkinson's disease (PD).

He said several proteins mutated in some hereditary forms of PD associate with lipid rafts, though the significance of these associations is unclear.

Schneider is the founder of LZ Therapeutics Inc., which is developing GM1 for PD.

"It's always been a question in PD and other neurodegenerative disorders-is there a systemic defect in lipid metabolism, and does this contribute to cell death?" said Schneider.

Schneider has evidence that GM1 can slow the progression of PD. In November, his team reported at the Society for Neuroscience conference the results of a six-month, double-blind Phase II trial of intraventricular GM1 in patients with PD followed by a two-year open-label extension. ${ }^{4}$

In that trial, GM1 given for six months significantly improved Unified Parkinson's Disease Rating Scale (UPDRS) motor function in patients compared with placebo. In the extension, all patients received GM1 and showed a slower rate of UPDRS decline than historical controls.

Schneider is now planning a larger Phase II PD trial for which he hopes to obtain NIH funding.

Meanwhile, LZ Therapeutics has licensed Schneider's patents on GM1 therapy for PD and is developing LZT-1000, a formulation of GM1, for PD and other neurological disorders. LZ Therapeutics CEO Jyrki Mattila said the company is scaling up production of LZT-1000 in preparation for its own clinical development of the compound in PD independently of Schneider's work.
Mattila said that LZ is looking to raise $\$ 20$ million in series B financing to develop LZT-1000 through Phase II testing.

Sipione has filed for a patent covering the use of GM1 in HD and is negotiating with an undisclosed biotech to advance the strategy into clinical development.

Osherovich, L. SciBX 5(10); doi:10.1038/scibx.2012.243

Published online March 8, 2012

\section{REFERENCES}

1. Di Pardo, A. et al. Proc. Natl. Acad. Sci. USA; published online Feb. 13, 2012; doi:10.1073/pnas.1114502109

Contact: Simonetta Sipione, University of Alberta, Edmonton, Alberta, Canada e-mail: ssipione@ualberta.ca

2. Maglione, V. et al. J. Neurosci. 30, 4072-4080 (2010)

3. Denny, C.A. et al. J. Neurochem. 115, 748-758 (2010)

4. Schneider, J.S. et al. Parkinson's disease: mechanisms of degeneration and treatment I. Presented at the Society for Neuroscience annual meeting, Nov. 12-16, 2011

\section{COMPANIES AND INSTITUTIONS MENTIONED} Gladstone Institute of Neurological Disease, San Francisco, Calif.

H. Lundbeck A/S (CSE:LUN), Copenhagen, Denmark LZ Therapeutics Inc., Malvern, $\mathrm{Pa}$. NeuroSearch A/S (CSE:NEUR), Ballerup, Denmark Society for Neuroscience, Washington, D.C. Thomas Jefferson University, Philadelphia, Pa. University of Alberta, Edmonton, Alberta, Canada University of California, San Francisco, Calif. 